\title{
TOXOPLASMA GONDII - SOROPREVALÊNCIA EM PACIENTES HIV NO SUL DO BRASIL
}

\author{
TOXOPLASMA GONDII - SEROPREVALENCE IN \\ HIV PATIENTS IN SOUTHERN BRAZIL
}

\author{
Claudinei Mesquita da Silva', Leyde Daiane de Peder², Rafael Andrade Menolli³, \\ Maria das Graças Marciano Hirata Takizawa4, Marcelo Caname Hirata Takizawa5, \\ Josana Dranka Horvath ${ }^{6}$, Eraldo Schunk Silva7, Jorge Juarez Vieira Teixeira ${ }^{8}$, Dennis Armando Bertolini9
}

\section{RESUMO}

O objetivo desse estudo foi estimar a soroprevalência de Toxoplasma gondii e sua associação com preditores em pacientes infectados pelo HIV. Estudo transversal realizado com pacientes infectados pelo HIV, atendidos em um centro de referência no Sul do Brasil, entre 2005 a 2014. A soroprevalência de T. gondii foi $57,90 \%$, sendo significativamente maior em pacientes pertencentes às faixas etárias de 30-49 anos (Odds Ratio (OR) 5,2; 95\% IC 1,57-17,10; p=0,007) e $\geq 50$ anos (OR 4,1; 95\% IC 1,22-13,77; $p=0,02$ ), sexo feminino (OR 3,3;95\% IC 1,33-7,32; $p<0,0001$ ) e com $\leq 8$ anos de educação (OR 2,2; IC $95 \% 1,62-2,93 ; p<0,0001)$. Pacientes solteiros e com tempo de diagnóstico do HIV $\leq 1$ ano possuíam fator de proteção (OR 0,5; $95 \%$ IC 0,25-0,99; p=0,04) e (OR 0,5; IC 0,37-0,73; p<0,0001), respectivamente. Os achados reportam alta soroprevalência para $T$. gondii em pacientes infectados com HIV.

Descritores: HIV; Toxoplasmose; Epidemiologia.

\section{ABSTRACT}

The objective of this study was to estimate the seroprevalence of Toxoplasma gondii and its association with predictors in HIV-infected patients. A cross-sectional study with HIV-infected patients attended at a referral center in Southern Brazil between 2005 and 2014. The seroprevalence of $T$. gondii was $57.90 \%$, being significantly higher in patients belonging to $30-49$ years (Odds Ratio (OR) $5.2,95 \% \mathrm{Cl}$ 1.57-17.10, $p=0.007$ ) and $\geq 50$ years (OR 4.1, 95\% Cl 1.22-13.77, $p=0,02$, female (OR $3.3,95 \% \mathrm{Cl}$ 1.33-7.32, $p<0.0001$ ) and with $\leq 8$ years of education (OR 2.2, 95\% Cl 1.62-2, 93, p <0.0001). Single patients with HIV diagnosis time $\leq 1$ year had a protective factor (OR $0.5,95 \% \mathrm{Cl} 0.25-0.99, \mathrm{p}=0.04)$ and (OR 0.5, $\mathrm{Cl} 0.37-0.73, \mathrm{p}<0.0001)$, respectively. The findings report high seroprevalence for $T$. gondii in HIV-infected patients.

Descriptors: HIV; Toxoplasmosis; Epidemiology.
${ }^{1}$ Doutorando em Ciências da Saúde pela Universidade Estadual de Maringá (UEM) Maringá, PR, Brasil.

${ }^{2}$ Doutoranda em Biociências e Fisiopatologia pela Universidade Estadual de Maringá (UEM), Maringá, PR, Brasil.

${ }^{3}$ Doutor em Biotecnologia pela Faculdade Pequeno Príncipe (FPP), Curitiba, PR, Brasil.

${ }^{4}$ Mestre em Ciências da Saúde pela Universidade Estadual de Maringá (UEM), Maringá, PR, Brasil.

${ }^{5}$ Pós-Graduação a nível de aperfeiçoamento em Endodontia pela Associação Brasileira de Odontologia (ABO) Cascavel, PR, Brasil.

${ }^{6}$ Especialista em Enfermagem e Infectologia no Instituto de Infectologia Emilio Ribas de São Paulo, São Paulo, SP, Brasil.

${ }^{7}$ Doutor em Agronomia pela Universidade Estadual Paulista Júlio de Mesquita Filho (UNESP), Botucatu, SP, Brasil.

${ }^{8}$ Doutor em Saúde Pública e Pós- Doutorado pela Universidade de São Paulo (USP), São Paulo, SP, Brasil.

${ }^{9}$ Doutor em Ciências pela Universidade Federal de São Paulo (UNIFESP), São Paulo, SP, Brasil. 


\section{Introdução}

A Toxoplasmose é a manifestação clínica de uma doença causada por Toxoplasma gondii, um protozoário intracelular obrigatório ${ }^{1,2}$. O protozoário possui ampla distribuição geográfica, afetando aproximadamente um terço da população mundial, na maioria das vezes de forma assintomática ${ }^{3}$. A prevalência da doença pode variar de 10 a $90 \%$ de acordo com os hábitos socioculturais, geográficos, climáticos, dentre outros ${ }^{3}$.

A infecção primária em indivíduos imunocompetentes é na maioria assintomática ou pode manifestar-se como linfadenopatia, sendo geralmente seguida por uma infecção latente ao longo da vida. Contudo, a partir deste estado de latência, a infecção por T. gondii pode ser reativada devido a perturbações imunitárias ${ }^{4}$. Apesar da disponibilidade de terapia antirretroviral (TARV), a toxoplasmose continua sendo a infecção oportunista mais nociva em pacientes infectados com 0 vírus da imunodeficiência humana (HIV) ${ }^{5,6}$. Nesses indivíduos, há aumento do risco da reativação da infecção latente por T. gondii em vários órgãos, particularmente no cérebro, o que pode conduzir à encefalite toxoplásmica ${ }^{7}$.

Em pacientes portadores da infecção, a reativação pode ocorrer de diversas formas, mas o mecanismo exato ainda não está bem esclarecido ${ }^{8}$. Em torno disso, pesquisas vêm demonstrando que células T CD4+ podem estar diretamente relacionadas com a resposta imunológica contra o protozoário, bem como com células T CD8+ e Natural Killer (NK). Deficiências nesses grupos celulares podem contribuir para a reativação da $T$. gondii ${ }^{8}$. Pacientes HIV positivos com toxoplasmose ativa apresentaram queda na contagem de células T CD4+ quando comparadas com os sem manifestações da toxoplasmose, e esse declínio pode estar relacionado com a progressão da infecção pelo HIV e com efeitos da infecção por T. gondii .

Tendo em conta a dimensão da infecção pelo HIV no Brasil|10,11,12 e a vulnerabilidade das pessoas infectadas pelo HIV com toxoplasmose, existe uma clara necessidade de mais estudos sobre este tema ${ }^{13,14}$. Além disso, o conhecimento da soroprevalência para $T$. gondii e dos fatores de risco associados em pessoas que vivem com HIV são essenciais para o melhor entendimento da dinâmica da infecção e, consequentemente, melhores alternativas para prevenção, manejo clínico e de tratamento ${ }^{15}$. Portanto, o objetivo deste estudo foi investigar a soroprevalência para T. gondii e sua associação com preditores em pacientes infectados pelo HIV, em um centro de referência de DST no Sul do Brasil.

\section{Metodologia}

Estudo de corte transversal realizado em um Centro de Referência para pacientes HIV/aids, pertencente à $10^{\mathrm{a}}$ Regional de Saúde do Estado do Paraná (10ª RS-PR), que atende 25 municípios. A população foi constituída por 1256 pacientes com diagnóstico positivo para o HIV, que tiveram acesso ao serviço no período de janeiro de 2005 a dezembro de 2014. Os dados sócio-demográficos, assim como as características laboratoriais foram obtidas dos prontuários médicos. Os pacientes que não possuíam residência fixa nos municípios pertencentes à $10^{\mathrm{a}} \mathrm{RS}$-PR foram excluídos.

Os exames sorológicos para diagnóstico do HIV foram realizados conforme as normas do Ministério da Saúde ${ }^{16}$. A sorologia para Toxoplasma gondii foi realizada utilizando kit comercial ELISA (IgM e IgG-Nova Lisa TM, Dietzenbach, Germany) de acordo com as instruções do fabricante, no Laboratório Municipal de Cascavel, Cascavel, PR. Os resultados referentes à contagem de células T CD4+, T CD8+ e a quantificação de carga viral para o HIV-1, foram realizados pelo Laboratório de Virologia Clínica da Universidade Estadual de Maringá, empregando para quantificação de linfócitos T, a técnica de Citometria de Fluxo (BD Trucount ${ }^{T M}$ Tubes), com o aparelho FACS Calibur (Becton-Dickinson, New Jersey, USA) e, para carga viral do HIV, utilizou-se o kit Abbott Real Time ${ }^{T M}$ HIV-1 (ABBOTT GmbH \& Co. KG, Wiesbaden, Alemanha), com o aparelho m2000rt (Abbott Laboratórios do Brasil Ltda, Divisão de Diagnósticos).

Os dados foram organizados e tabulados no programa Microsoft Exce ${ }^{\circledR} \mathrm{e}$, posteriormente, analisados no software SAS (Statistical Analysis Software), versão 9.4. Os resultados foram expressos em médias, desvios-padrão ( \pm DP) ou frequências. A associação entre as variáveis qualitativas foi verificada por meio dos testes Qui-Quadrado ou Exato de Fisher. As variáveis quantitativas foram analisadas pelo Teste de Wilcoxon. Para a medida da estimativa do risco foi utilizado o Odds Ratio (OR). Modelos de regressão logística binária e multinomial foram ajustados para cada uma das covariáveis e eventos de interesse. Somente as variáveis que apresentaram valor de $p \leq 0,20$ foram mantidas no modelo. A significância estatística foi estabelecida para $p<0,05$. 0 estudo foi aprovado pelo Comitê de Ética em Pesquisa do Centro Universitário da Fundação Assis Gurgacz (Parecer nº 1.397.212 de 28 de janeiro de 2016). 


\section{Resultados}

Do total de 1256 pacientes atendidos e diagnosticados com HIV no período analisado, 1186 (94.42\%) eram residentes na região de estudo, sendo que desses, $772(65,09 \%)$ realizaram testes sorológicos para detecção de $T$. gondii. A prevalência para anticorpos (lgG e $\operatorname{lgM})$ anti-T. gondii foi de 57,90\% (447/772), sendo que destes, $223(49,89 \%)$

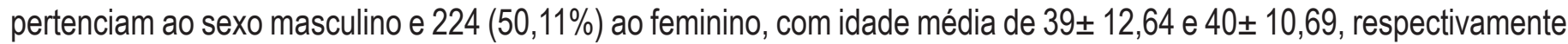
$(p>0,05)$. A maior soroprevalência para T. gondii foi reportada na faixa etária entre 30-49 anos $(63,09 \%)(p<0,05)$. Pacientes casados também apresentaram maior soroprevalência para T. gondii (44,94\%), assim como, pacientes com tempo de educação menor ou igual a 8 anos $(65,29 \%)$, heterossexuais $(88,69 \%)$ e com tempo de diagnóstico do HIV maior que 5 anos $(48,55 \%)$, todas com significância estatística $(p<0,05)$ (Tabela 1).

Tabela 1 - Características sócio demográficas de pacientes com HIV, de acordo com a sorologia para toxoplasmose, 10 RS-PR, Brasil, 2005-2014

\begin{tabular}{|c|c|c|c|c|}
\hline $\begin{array}{l}\text { Variáveis } \\
\text { preditoras }\end{array}$ & $\begin{array}{l}\text { Total } \\
\text { n (\%) }\end{array}$ & $\begin{array}{c}\text { Sorologia } \\
\text { negativa para } \\
\text { toxoplasmose } \\
\text { n (\%) }\end{array}$ & $\begin{array}{c}\text { Sorologia } \\
\text { positiva para } \\
\text { toxoplasmose } \\
\text { n (\%) }\end{array}$ & p-valor \\
\hline \multicolumn{5}{|l|}{ Idade (anos) } \\
\hline $0-17$ & $14(1,82)$ & $10(3,11)$ & $4(0,89)$ & \multirow{4}{*}{$0.005 \neq$} \\
\hline $18-29$ & $169(21,98)$ & $90(27,95)$ & $79(17,67)$ & \\
\hline $30-49$ & $454(59,04)$ & $172(53,42)$ & $282(63,09)$ & \\
\hline$\geq 50$ & $132(17,16)$ & $50(15,52)$ & $82(18,34)$ & \\
\hline \multicolumn{5}{|l|}{ Sexo } \\
\hline Masculino & $411(53,45)$ & $188(58,39)$ & $223(49,89)$ & \multirow{2}{*}{$0,024 \neq$} \\
\hline Feminino & $358(46,55)$ & $134(41,61)$ & $224(50,11)$ & \\
\hline \multicolumn{5}{|l|}{ Estado civil } \\
\hline Solteiro & $339(44,55)$ & $164(51,90)$ & $175(39,33)$ & \multirow{4}{*}{$0.007 \neq$} \\
\hline Casado & $316(41,52)$ & $116(36,71)$ & $200(44,94)$ & \\
\hline Divorciado & $65(8,54)$ & $23(7,28)$ & $42(9,44)$ & \\
\hline Viúvo & $41(5,39)$ & $13(4,11)$ & $28(6,29)$ & \\
\hline \multicolumn{5}{|l|}{ Etnia } \\
\hline Branca & $504(66,06)$ & $226(70,63)$ & $278(62,75)$ & \multirow{3}{*}{0,052} \\
\hline Pardo & $234(30,67)$ & $87(27,19)$ & $147(33,18)$ & \\
\hline Outras & $25(3,27)$ & $7(2,19)$ & $18(4,06)$ & \\
\hline \multicolumn{5}{|c|}{ Educação (anos) } \\
\hline$\leq 8$ & $430(57,33)$ & $146(46,35)$ & $284(65,29)$ & \multirow{2}{*}{$<0,0001 \neq$} \\
\hline$>8$ & $320(42,67)$ & $169(53,65)$ & $151(34,71)$ & \\
\hline \multicolumn{5}{|l|}{ Comportamento } \\
\hline Bissexual & $34(4,50)$ & $14(4,47)$ & $20(4,52)$ & \multirow{3}{*}{$<0,0001 \neq$} \\
\hline Heterossexual & $624(82,65)$ & $232(74,12)$ & $392(88,69)$ & \\
\hline Homossexual & $97(12,85)$ & $67(21,41)$ & $30(6,79)$ & \\
\hline
\end{tabular}

Continua... 


\begin{tabular}{|c|c|c|c|c|}
\hline $\begin{array}{l}\text { Variáveis } \\
\text { preditoras }\end{array}$ & $\begin{array}{l}\text { Total } \\
\text { n (\%) }\end{array}$ & $\begin{array}{c}\text { Sorologia } \\
\text { negativa para } \\
\text { toxoplasmose } \\
\text { n (\%) }\end{array}$ & $\begin{array}{c}\text { Sorologia } \\
\text { positiva para } \\
\text { toxoplasmose } \\
\mathbf{n}(\%)\end{array}$ & p-valor \\
\hline \multicolumn{5}{|c|}{ Residência/cidade (habitantes) } \\
\hline $\begin{array}{c}\text { População } \leq \\
10.000\end{array}$ & $62(8,06)$ & $22(6,83)$ & $40(8,95)$ & \multirow{3}{*}{0,256} \\
\hline $\begin{array}{c}\text { População > } \\
10.000 \\
\end{array}$ & $138(17,95)$ & $52(16,15)$ & $86(19,24)$ & \\
\hline Cascavel & $569(73,99)$ & $248(77,02)$ & $321(71,81)$ & \\
\hline \multicolumn{5}{|c|}{ Tempo do diagnóstico do HIV (anos) } \\
\hline$\leq 2$ & $226(29,39)$ & $119(36,96)$ & $107(23,94)$ & \multirow{3}{*}{$0,001 \neq$} \\
\hline $2-5$ & $201(26,14)$ & $78(24,22)$ & $123(27,52)$ & \\
\hline$>5$ & $342(44,47)$ & $125(38,82)$ & $217(48,55)$ & \\
\hline \multicolumn{5}{|c|}{ Número de parceiros sexuais últimos 12 meses } \\
\hline$\leq 1$ & $391(58,01)$ & $146(53,68)$ & $245(60,95)$ & \multirow{3}{*}{0,104} \\
\hline $2-5$ & $101(14,99)$ & $41(15,07)$ & $60(14,93)$ & \\
\hline$>5$ & $182(27,00)$ & $85(31,25)$ & $97(24,13)$ & \\
\hline \multicolumn{5}{|c|}{ Usuários de drogas } \\
\hline Sim & $77(10,52)$ & $36(12,08)$ & $41(9,45)$ & \multirow{2}{*}{0,308} \\
\hline Não & $655(89,48)$ & $262(87,92)$ & $393(90,55)$ & \\
\hline \multicolumn{5}{|c|}{ Usuários de drogas injetáveis } \\
\hline Sim & $8(1,09)$ & $2(0,66)$ & $6(1,39)$ & \multirow{2}{*}{0,350} \\
\hline Não & $726(98,91)$ & $299(99,34)$ & $427(98,61)$ & \\
\hline
\end{tabular}

HIV, vírus da imunodeficiência humana; n, número de pacientes; teste exato de Fisher ou teste do Quiquadrado de Pearson para a comparação entre os grupos; \# Diferença estatisticamente significativa p<0,05.

Do total de pacientes infectados pelo HIV, 545 pacientes (324 com sorologia positiva e 221 com sorologia negativa para T. gondii) possuíam dados referentes às contagens de células $T$ CD4+, relação $C D 4 / C D 8$ e estavam em uso da TARV. A maioria, tanto com sorologia positiva, quanto negativa, possuía contagem média de células T CD4+ maior que 500 células $/ \mathrm{mm}^{3}$. Não houve associação estatística nas contagens de células T CD4+ e relação CD4/CD8 nos grupos HIV com sorologia positiva e negativa para T. gondii $(p>0,05)$ (Tabela 2 ).

Tabela 2 - Níveis de células T CD4+ e relação CD4/CD8 em pacientes portadores de HIV, com sorologia positiva e negativa para toxoplasmose, 10 RS-PR, Brasil, 2005-2014.

\begin{tabular}{|c|c|c|c|c|c|c|c|}
\hline \multirow[b]{2}{*}{ Grupos } & \multirow{2}{*}{$\begin{array}{c}\text { Média } \\
\text { Relação } \\
\text { CD4/CD8 }\end{array}$} & \multirow[b]{2}{*}{ p-valor } & \multicolumn{5}{|c|}{ Contagens de células T CD4+ (células/mm³) } \\
\hline & & & $\begin{array}{l}\text { Total } \\
\text { n (\%) }\end{array}$ & $\begin{array}{l}\leq 200 \\
\mathrm{n}(\%)\end{array}$ & $\begin{array}{c}200-500 \\
n(\%)\end{array}$ & $\begin{array}{l}>500 \\
\mathrm{n}(\%)\end{array}$ & p-valor \\
\hline $\begin{array}{l}\text { HIV-sorologia } \\
\text { positiva para } \\
\text { toxoplasmose }\end{array}$ & $0,60 \pm 0,36$ & 0,10 & $221(100)$ & $20(9,05)$ & $69(31,22)$ & $132(59,73)$ & 0,26 \\
\hline $\begin{array}{l}\text { HIV-sorologia } \\
\text { negativa para } \\
\text { toxoplasmose }\end{array}$ & $0,63 \pm 0,42$ & & $324(100)$ & $19(5,87)$ & $94(29,01)$ & $211(65,12)$ & \\
\hline
\end{tabular}

HIV, o vírus da imunodeficiência humana; $n$, número de pacientes;

Qui-quadrado de Pearson para a comparação entre os grupos. 
Fatores de risco para infecção para T. gondii em pacientes infectados pelo HIV foram analisados por regressão logística multinomial. Pacientes infectados pelo HIV do sexo feminino possuíam maior chance de ter sorologia positiva para T. gondii em comparação com os homens (OR 3,3; IC 95\% 1.33-7.23; $p<0,0001$ ), assim como, pacientes com $\leq 8$ anos de educação (OR 2,2; IC 95\% 1,62-2,93; $p<0,0001$ ) e pacientes pertencentes às faixas etárias entre 30-49 anos e $\geq 50$ anos (OR 5,2; IC 95\% 1,57-17,10; $p=0,07$ ) e (OR 4,1; IC 95\% 1,22-13,77; p=0,02), respectivamente. Pacientes que possuíam tempo de diagnóstico do HIV $\leq 1$ ano e solteiros possuíam fator de proteção para T. gondii (OR 0,5; 95\% IC $0,37-0,73 ; p<0,0001)$ e (OR 0,5; 95\% IC 0,25-0,99; $p=0,04)$, respectivamente (Tabela 3).

Tabela 3 - Análise logística multivariada das variáveis preditoras com sorologia positiva

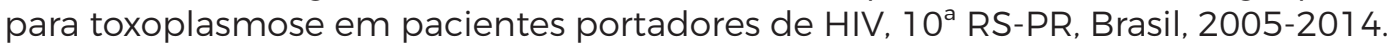

\begin{tabular}{|c|c|c|c|}
\hline \multicolumn{4}{|c|}{ HIV-T. gondii } \\
\hline Características & OR & 95\% C.I & p-valor \\
\hline \multicolumn{4}{|l|}{ Idade (anos) } \\
\hline$\leq 17$ & 1 & - & - \\
\hline $18-29$ & 2,2 & $0,66-7,27$ & 0,20 \\
\hline $30-49$ & 5,2 & $1,57-17,10$ & $0,007 \neq$ \\
\hline$\geq 50$ & 4,1 & $1,22-13,77$ & $0,02 \neq$ \\
\hline \multicolumn{4}{|l|}{ Sexo } \\
\hline Masculino & 3,3 & $1,33-7,32$ & $<0,0001 \neq$ \\
\hline Feminino & 1 & - & - \\
\hline \multicolumn{4}{|l|}{ Estado civil } \\
\hline Solteiro & 0,5 & $0,25-0,99$ & $0,04 \neq$ \\
\hline Casado & 0,8 & $0,40-1,61$ & 0,53 \\
\hline Divorciado & 0,8 & $0,37-1,95$ & 0,69 \\
\hline Viúvo & 1 & - & - \\
\hline
\end{tabular}

Educação (anos)

\begin{tabular}{c|c|c|c}
\hline$\leq 8$ & 2,2 & $1,62-2,93$ & $<0,0001 \neq$ \\
\hline$>8$ & 1 & - & - \\
\hline Comportamento & \multicolumn{5}{|c}{} \\
\hline Homossexual & 0,3 & $0,14-0,70$ & $0,005 \neq$ \\
\hline Bissexual & 1 & - & - \\
\hline Heterossexual & 1,2 & $0,59-2,39$ & 0,30 \\
\hline
\end{tabular}

Tempo do diagnóstico do HIV (anos)

\begin{tabular}{c|c|c|c}
\hline$\leq 1$ & 0,5 & $0,37-0,73$ & $<0,0001 \neq$ \\
\hline $2-5$ & 0,9 & $0,63-1,30$ & 0,60 \\
\hline$>5$ & 1 & - & - \\
\hline
\end{tabular}

HIV, vírus da imunodeficiência humana; n, número de pacientes; OR, razão de chances; $\mathrm{Cl}$, intervalo de confiança; $\neq$ Diferença estatisticamente significativa, $\mathrm{p}<0,05$. 


\section{Discussão}

A vigilância da infecção por T. gondii é sugerida em pacientes infectados pelo HIV ${ }^{17}$ e a reativação da infecção latente por Toxoplasma é comum em hospedeiros imunocomprometidos, o que torna os pacientes HIV com maior risco de apresentar toxoplasmose clínica ${ }^{18}$. Aproximadamente metade dos pacientes infectados pelo HIV são coinfectados com T. gondii ${ }^{19,20}$, no entanto, as soroprevalências apresentam variações ${ }^{3}$. A soroprevalência observada no presente estudo $(57,90 \%)$ foi maior em relação à encontrada na Tailândia $(36,3 \%)^{18}$, em Moçambique $(46 \%)^{14}$ e nos Estados Unidos $(15,68 \%)^{21}$ e menor quando comparada a estudos realizados no Brasil, especificamente em Minas Gerais, Rio Grande do Sul e São Paulo que apresentaram soroprevalência de $80 \%, 78 \%$ e $91 \%$, respectivamente ${ }^{15,22,23}$. Essas diferenças provavelmente são devidas aos hábitos alimentares, variações geográficas, condições de saneamento básico, diferenças sócio culturais e hábitos de vida ${ }^{18}$. Assim, fatores de risco previamente identificados, como consumo de alimentos possivelmente contaminados podem estar associados com a infecção por T. gondii ${ }^{18}$.

Em relação ao sexo, praticamente metade $(50,11 \%)$ dos pacientes com sorologia positiva para $T$. gondii eram mulheres. Utilizando modelos de regressão logística binária e multinomial, o sexo feminino foi fator de risco para infecção por T. gondii (OR 3,3; IC 95\% 1,33-7,32; p<0,0001). Outros estudos reportam que as mulheres infectadas pelo HIV foram as mais acometidas pela infecção por T. gondii' ${ }^{14,17}$. Tal achado pode estar associado à ativação do cisto ou pseudocisto, que é facilmente induzido pelo alto nível de estrogênio ${ }^{24}$.

Especificamente, a maior prevalência de T. gondii foi encontrada também em pacientes com idades compreendidas entre 30 e 49 anos (282/63,09\%). Um estudo mostrou que episódios de retinocoroidite toxoplasmática foram relatados serem mais frequentes em pacientes com 31,1 anos, isto é, mais de $82 \%$ dos casos de retinocoroidite toxoplasmática (71 de 87 episódios) de todos os episódios observados e ocorreram principalmente em pacientes de 15 a 44 anos de idade ${ }^{25}$. Além disso, em 2002, Bosch-Driessen et al. relataram que mais de 78\% (215 de 274 episódios) de episódios de Toxoplasmose ocular ativa ocorreram em pacientes com idade entre 15 e 45 anos $^{26}$. Utilizando a análise multivariada, este estudo identificou que pacientes HIV com faixa etária de 30-49 anos tem 5,2 vezes mais chances de ter toxoplasmose (OR 5,2; IC 1,47-17,10; $p=0,007$ ), assim como pacientes com idade maior/igual a 50 anos (OR 4,1; IC 1,33-7,32; $p=0,02$ ). A estratificação da idade baseada em prevalência destaca a importância da idade do paciente como um possível fator de risco, particularmente em adultos mais jovens e de meia-idade ${ }^{27}$.

Em contextos de escassez de recursos, qualquer associação entre soropositividade ao $T$. gondii e variáveis clínicas relacionadas ao HIV, como contagem de células T CD4+, pode ser útil na classificação de pacientes que podem se beneficiar do rastreio da infecção ou da profilaxia contra toxoplasmose ${ }^{28}$. O monitoramento da infecção por $T$. gondii, principalmente, com base nas contagens de células T CD4+ ajuda a retardar a progressão da doença, uma vez que as funções imunitárias dos pacientes infectados pelo HIV são progressivamente prejudicadas ${ }^{17}$. As células T CD4+ são críticas na defesa imunológica contra a toxoplasmose em pacientes infectados pelo HIV e a reativação da infecção por T. gondii está associada ao declínio desse subconjunto de células $T^{29}$. No presente estudo, a maioria dos pacientes tanto com sorologia positiva quanto com negativa para T. gondii possuía contagem de células T CD4+ maior que 500 células/ $\mathrm{mm}^{3}$ e a soropositividade para o parasita não estava associada às contagens de células T CD4+. Estudos no México ${ }^{30}$ e Malásia ${ }^{31}$ também não mostraram correlação entre a contagem de células T CD4+ e soropositividade para T. gondii.

O estudo possui algumas limitações, já que não foi possível determinar a causa soropositividade de anticorpos anti-T. gondii na população HIV estudada e também pelo estudo ter sido realizado em um centro de referência para HIV e não em um ambiente comunitário. Dessa forma, a pesquisa ainda está sujeita à presença de viés de informação, devido à utilização de fonte secundária, embora os resultados sejam consistentes com outros estudos e são relevantes para planejar o atendimento clínico de pacientes com infecção pelo HIV.

\section{Considerações Finais}

Os resultados deste estudo demonstram uma soroprevalência de $57,90 \%$ para infecção por $T$. gondii em pacientes HIV. Contagem de células T CD4+ e relação CD4/CD8 não foram significativamente associados com a sorologia para T. gondii. Faixa etária maior/igual a 30 anos, sexo feminino, solteiros, foram identificados como os principais preditores para soroprevalência positiva para T. gondii em pacientes HIV. Recomenda-se a educação preventiva de rotina para toxoplasmose em pacientes infectados pelo HIV em nossa região, assim como, naquelas com alta soropositividade, bem 
como rastreio de rotina de todos os pacientes infectados pelo HIV para anticorpos IgG anti-Toxoplasma, para identificar pacientes em risco de encefalite Toxoplásmica. Esta medida poderia reduzir impactos clínicos e de saúde pública. Como parte deste esforço, a integração do serviço de triagem e tratamento da toxoplasmose com o tratamento do HIV é necessária e poderia limitar as consequências clínicas da toxoplasmose não tratada.

\section{Referências}

1. Dubey JP, Jones JL. Toxoplasma gondii infection in humans and animals in the United States. Int J Parasitol. 2008;8:1257-78.

2. Hill DE, Chirukandoth S, Dubey JP. Biology and epidemiology of Toxoplasma gondii in man and animals. Anim Heal Res Rev. 2005;6(1):41-61.

3. Montoya JG, Liesenfeld O. Toxoplasmosis. Lancet (London, England). 2004;363(9425):1965-76.

4. Meers S, Lagrou K, Theunissen K, Dierickx D, Delforge M, Devos T, et al. Myeloablative conditioning predisposes patients for Toxoplasma gondii reactivation after allogeneic stem cell transplantation. Clin Infect Dis. 2010;50(8):1127-34. 5. Abgrall S, Rabaud C, Costagliola D. Incidence and risk factors for toxoplasmic encephalitis in human immunodeficiency virus-infected patients before and during the highly active antiretroviral therapy era. Clin Infect Dis. 2001;33(10):1747-55. 6. Israelski DM, Chmiel JS, Poggensee L, Phair JP, Remington JS. Prevalence of Toxoplasma infection in a cohort of homosexual men at risk of AIDS and toxoplasmic encephalitis. J Acquir Immune Defic Syndr. 1993;6(4):414-8.

7. Sukthana Y, Chintana T, Lekkla A. Toxoplasma gondii Antibody in HIV-Infected Persons. J Med Assoc Thail. 2000;83(6):681-4.

8. Bhadra R, Khan IA. Redefining Chronic Toxoplasmosis-A T Cell Exhaustion Perspective. PLoS Pathog. 2012;8(10):8-11.

9. Beran O, Kodym P, Maly M, Davidova A, Reinvartova G, Jilich D, et al. The Effect of Latent Toxoplasma gondii Infection on the Immune Response in HIV-Infected Patients. Biomed Res Int. 2015;2015:1-7.

10. Kilmarx PH. Global epidemiology of HIV. Curr Opin HIV AIDS. 2009;4(4):240-6.

11. de Souza SMB, Teles SA, Rezza G, Pezzotti P, Gir E. Epidemiology of HIV infection in central Brazil: data from voluntary counseling and testing centers. J Assoc Nurses AIDS Care. 2013;24(6):503-11.

12. Librelotto CS, Graf T, Simon D, de Almeida SEM, Lunge VR. HIV-1 epidemiology and circulating subtypes in the countryside of South Brazil. Rev Soc Bras Med Trop. 2015;48(3):249-57.

13. Lindström I, Kaddu-Mulindwa DH, Kironde F, Lindh J. Prevalence of latent and reactivated Toxoplasma gondii parasites in HIV-patients from Uganda. Acta Trop. 2006;100(3):218-22.

14. Domingos A, Ito LS, Coelho E, Lúcio JM, Matida LH, Ramos AN. Seroprevalence of Toxoplasma gondii lgG antibody in HIVIAIDS-infected individuals in Maputo, Mozambique. Rev Saude Publica. 2013;47(5):890-6.

15. Joice Silva Alves, Vinicius Silva Belo, Renata Coelho de Castro ES da S. Factors associated with soroprevalence of antibodies IgG and IgM anti-toxoplasma in HIVIAIDS patientes assisted in an expert assistance service. J Heal Biol Sci. 2016;4(3):145-51.

16. BRASIL. Ministério da Saúde. Secretaria de Vigilância em Saúde. Departamento de DST, Aids e Hepatites Virais. Manual Técnico para o Diagnóstico da Infecção pelo HIV, 2013.

17. Shen G, Wang X, Sun H, Gao Y. Seroprevalence of Toxoplasma gondii Infection among HIVIAIDS Patients in Eastern China. Korean J Parasitol. 2016;54(1):93-6.

18. Chemoh W, Sawangjaroen N, Siripaitoon P, Andiappan H, Hortiwakul T, Sermwittayawong N, et al. Toxoplasma gondii Prevalence and risk factors in HIV-infected patients from Songklanagarind Hospital, Southern Thailand. Front Microbiol. 2015;6:1304 19. Shimelis T, Tebeje M, Tadesse E, Tegbaru B, Terefe A. Sero-prevalence of latent Toxoplasma gondii infection among HIVinfected and HIV-uninfected people in Addis Ababa, Ethiopia: A comparative cross-sectional study. BMC Res Notes. 2009;2:213. 20. Daryani A, Sharif M, Meigouni M. Seroprevalence of lgG and IgM anti-Toxoplasma antibodies in HIVIAIDS patients, northern Iran. Asian Pac J Trop Med. 2011;4(4):271-4.

21. O'Bryan TA, Okulicz JF, Bradley WP, Ganesan A, Merritt SE, Agan BK. Toxoplasma gondii seroprevalence: 30-year trend in an HIV-infected US military cohort. Diagn Microbiol Infect Dis. 2016;84(1):34-5.

22. Xavier GA, Cademartori BG, Cunha Filho NA da, Farias NA da R. Evaluation of seroepidemiological toxoplasmosis in HIVIAIDS patients in the south of Brazil. Rev Inst Med Trop São Paulo. 2013;55(1):25-30. 
23. Vidal JE, Hernández Diaz AV, de Oliveira ACP, Dauar RF, Colombo FA, Pereira-Chioccola VL. Importance of high IgG anti-Toxoplasma gondii titers and PCR detection of T. gondii DNA in peripheral blood samples for the diagnosis of AIDSrelated cerebral toxoplasmosis: A case-control study. Brazilian J Infect Dis. 2011;15(4):356-9.

24. Xiao Y, Yin J, Jiang N, Xiang M, Hao L, Lu H. Seroepidemiology of human Toxoplasma gondii infection in China. BMC Infect Dis. 2010;10(1):4.

25. Gilbert RE, Dunn DT, Lightman S, Murray PI, Pavesio CE, Gormley PD. Incidence of symptomatic toxoplasma eye disease: aetiology and public health implications. Epidemiol Infect. 1999;123(2):283-9.

26. Bosch-Driessen LEH, Berendschot TTJM, Ongkosuwito J V., Rothova A. Ocular toxoplasmosis: Clinical features and prognosis of 154 patients. Ophthalmology. 2002;109(5):869-78.

27. Bustillo JL, Diaz JD, Pacheco IC, Gritz DC. Cuban Ocular Toxoplasmosis Epidemiology Study (COTES): incidence and prevalence of ocular toxoplasmosis in Central Cuba. Br J Ophthalmol. 2015;99(3):382-6.

28. Ogoina D, Onyemelukwe GC, Musa BO, Obiako RO. Seroprevalence of IgM and $\lg G$ antibodies to Toxoplasma infection in healthy and HIV-positive adults from Northern Nigeria. J Infect Dev Ctries. 2013;7(5):398-403.

29. Luft BJ, Brooks RG, Conley FK, McCabe RE, Remington JS. Toxoplasmic encephalitis in patients with acquired immune deficiency syndrome. Jama. 1984;252(7):913-7.

30. Gongora-Biachi RA, Gonzalez-Martinez P, Castro-Sansores C, Alvarez-Moguel R, Pavia-Ruz N, Lara-Perera D. Antibodies against Toxoplasma gondii in patients with HIV in Yucatan. Rev Invest Clin. 1998;50(5):419-22.

31. Nissapatorn V, Kamarulzaman A, Init I, Tan LH, Rohela M, Norliza A. Seroepidemiology of toxoplasmosis among HIVinfected patients and healthy blood donors. Med J Malaysia. 2002;57(3):304-10.

\section{Claudinei Mesquita da Silva}

Endereço para correspondência - Laboratório de Análises Clínicas, Centro Universitário da Fundação Assis Gurgacz, $n^{\circ}$ 500, Bairro FAG, CEP 85806-095, Cascavel, PR, Brasil.

E-mail: claudinei@fag.edu.br

Lattes: http://lattes.cnpq.br/3666201093308863

Leyde Daiane de Peder - leydepeder@yahoo.com.br

Rafael Andrade Menolli - ramenolli@hotmail.com

Maria das Graças Marciano Hirata Takizawa - graça@fag.edu.br

Marcelo Caname Hirata Takizawa - marcelotakizawa123@gmail.com

Josana Dranka Horvath - josanad@cascavel.pr.gov.br

Eraldo Schunk Silva - eraldoschunk@gmail.com

Jorge Juarez Vieira Teixeira - jjvteixeira@gmail.com

Dennis Armando Bertolini - dabertolini18@gmail.com

\section{Enviado em 12 de janeiro de 2017. Aceito em 23 de junho de 2017.}

